\title{
MEDIDAS DE CALIDAD DE CIELO (SEEING) USANDO LA TÉCNICA DIMM EN EL OBSERVATORIO DE HUANCAYO
}

\section{QUALITY MEASURES OF HEAVEN (SEEING) USING THE TECHNIQUE DIMMAT THE HUANCAYO OBSERVATORY}

\author{
Erick Meza ${ }^{1}$, Antonio Pereyra ${ }^{2}$, Bruno Sicardy $^{3}$, Germán Comina ${ }^{4}$, José Ishitsuka ${ }^{5}$ \\ RESUMEN
}

\begin{abstract}
Mostramos la implementación de un instrumento de medición de la calidad de cielo (seeing) basado en la técnica del Monitoreo del Movimiento Diferencial de Imagen (DIMM). Este instrumento permite la medición precisa y sistemática del seeing, así como su evolución temporal. Fue monitoreado el seeing en el Observatorio de Huancayo durante siete noches consecutivas de Agosto de 2012. El seeing promedio fue de 1.34” \pm 0.33”. En 14\% del tiempo observado el seeing estuvo por debajo de 1", y en $71 \%$ del tiempo, por debajo de 1.5". El dominio de la técnica y su aplicabilidad nos permitirá usar el instrumento en varios lugares donde campañas de medición de seeing sean programadas dentro del Perú. La selección de una facilidad astronómica óptica profesional (observatorio astronómico), será favorecida con medidas DIMM futuras.
\end{abstract}

Palabras clave.- Medición de la calidad de cielo, Diferencial de imagen (DIMM), Observatorio de Huancayo.

\section{ABSTRACT}

We show the implementation of an instrument to measure the sky quality for astronomical purposes (seeing) using the Differential Image Motion Monitor technique. This instrument let us an acute and systematic measurement of seeing along with its temporal evolution. We monitored seeing at Huancayo Observatory during seven consecutive nights in 2012 August. Mean seeing was 1.34" \pm 0.33 ". In 14\% of the observed time, seeing was below than 1", and in $71 \%$ below than 1.5". The successful implementation of this technique and its applicability let us using this instrument in several places where a program of site testing will be scheduled in Peru. The selection of a professional astronomical facility (as an astronomical observatory) will be benefited with DIMM measurements.

Key words.- Measuring qualityofsky, Differential Image (DIMM), Huancayo observatory.

\section{INTRODUCCIÓN}

La turbulencia atmosférica es uno de los mayores problemas en la astronomía en el óptico especialmente en la manera como reduce la resolución angular de los telescopios. El diámetro de la imagen de una estrella, también llamado disco de seeing, varía aproximadamente de 0.3 " a 10 " o más, de acuerdo a las condiciones meteorológicas. Cuando una imagen estelar con alta magnificación es observada a través del,

${ }^{1}$ Grupo Astronomía, Facultad de Ciencias, UNI , ${ }^{2}$ Instituto de Astrofísica de Canarias, Tenerife, España, ${ }^{3}$ LESIA, Observatorio de Paris, Francia, ${ }^{4}$ Laboratorio de Ingeniería Física, Facultad de Ciencias, UNI, "Área de Astronomía y Astrofísica, Instituto Geofísico del Perú. 
la estructura de la imagen observada, incluso con la óptica perfecta, está por lo general lejos del patrón de difracción teórico y cambia rápidamente con el tiempo. La forma de la imagen depende de la apertura del telescopio. Por lo general un movimiento aleatorio de la imagen es el principal efecto en telescopios de pequeña apertura. Con telescopios de apertura grande ocurre una difusión y desenfoque de la imagen. Frecuentemente una estructura moteada o "speckles", Rösch lo describe como un racimo de uva [1]. A inicio de los sesenta, la física de la degradación de la imagen era todavía no muy comprendida. Muchas técnicas en bases empíricas fueron elaboradas con el objetivo de realizar estimaciones cuantitativas de la degradación de la imagen $[2 \mathrm{y}, 3]$.

Actualmente la degradación de la imagen en la atmósfera terrestre es teóricamente bien entendida usando como base al modelo de turbulencia de Kolmogorov [4 y 5]. En este modelo, un solo parámetro, el parámetro de Fried $r_{0}[6]$ es suficiente para describir todos los efectos de la turbulencia "aveces llamado seeing" [7]. Este parámetro se puede medir a partir del movimiento de la imagen en un pequeño telescopio. En los telescopios modernos de última generación, es de extrema importancia el conocimiento de la razón de degradación, $\mathrm{D} / \mathrm{r}_{0}$. Por ejemplo, para un telescopio de $8 \mathrm{~m}$ de apertura y un valor típico $\mathrm{r}_{0}$ de $10 \mathrm{~cm}$, la degradación alcanza un factor 80. Esto tiene muchas repercusiones en diferentes campos de la astronomía como fotometría, proyección de imagen clásica (imaging), espectroscopia, etc. [8 y 9].

Muchos intentos diferentes han sido hechos para medir el seeing desde los trabajos pioneros de [1]. A pesar que los parámetros relevantes a la degradación de la imagen están relacionados a la geofísica (fluctuaciones turbulentas de la densidad del aire), la totalidad de los instrumentos de medición de seeingson instrumentos ópticos. Diferentes conceptos han sido usados, entre los cuales podemos destacar: la técnica Scidar[10], la técnica DIMM [2 y 11]; y la técnica GSM [12]. Las diferentes técnicas usan diferentes tipos de detectores que van desde el ojo humano, placas fotográficas, fotomultiplicadoras hasta los actuales CCDs. Los dispositivos tipo
DIMM son actualmente los instrumentos de tipo patrón para las mediciones de seeing.

\section{DIMM}

Un Monitor de Movimiento Diferencial de Imagen DIMM (por su sigla en inglés, DifferentialImageMotion Monitor) [8] es un sistema óptico formado por un telescopio en el cual la luz pasa por dos aperturas idénticas separadas una distancia $d$ como se observa en la Fig. 1. El principio es producir dos imágenes gemelas de una estrella, con el mismo telescopio. El cono de luz producido por cada apertura es desplazado por el prisma situado en una de ellas formando una imagen bien separada en el plano focal del telescopio. El movimiento relativo de ambas imágenes en el plano imagen representa inclinaciones locales del frente de onda.

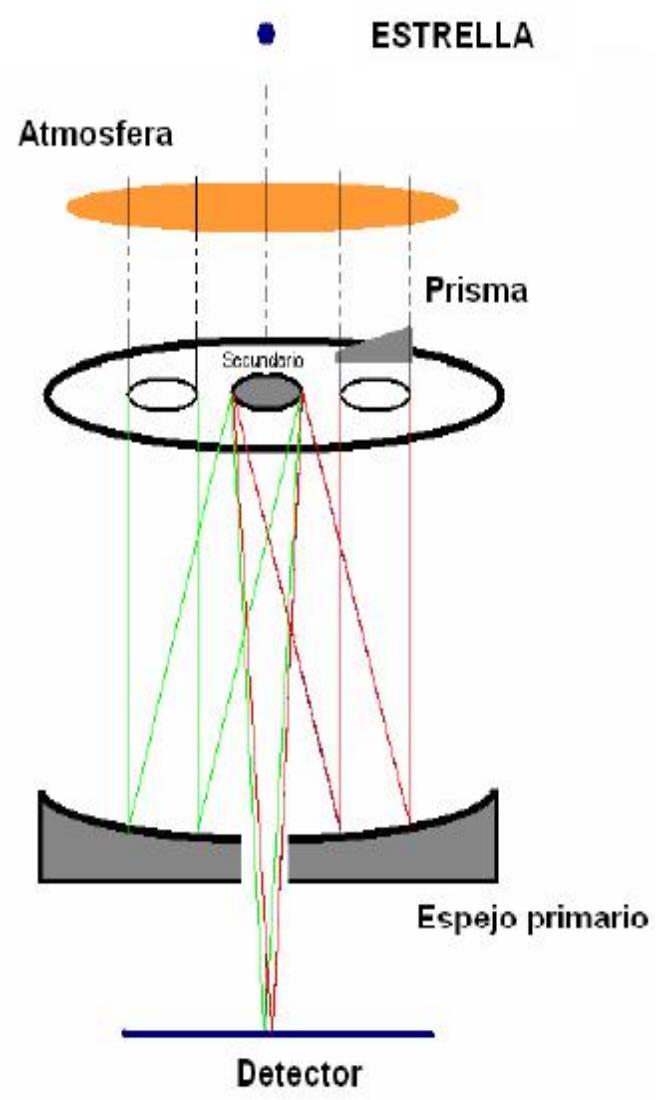

Fig. 1 Esquema óptico del OA - UNI DIMM. 
Existen factores que afectan por igual a las imágenes producidas por ambas aperturas, por ejemplo, las vibraciones del telescopio, sin embargo, hay otros factores que sólo afectan de forma diferente a cada una de las aperturas, como por ejemplo, la turbulencia atmosférica.

Estas pequeñas variaciones en la separación de las imágenes se utilizan para obtener una estimación cuantitativa de la turbulencia inducida por la fluctuación de fase del frente de onda. Este método diferencial elimina posibles movimientos erráticos del telescopio.

La varianza del movimiento longitudinal $\left(\sigma_{l}^{2}\right)$ y transversal $\left(\sigma_{t}^{2}\right)$ de una imagen diferencial viene dada por[11]:

$$
\begin{aligned}
\sigma_{l}^{2} & =2 \lambda^{2} r_{0}^{-5 / 3}\left[0.179 D^{-1 / 3}-0.0968 d^{-1 / 3}\right] \\
\sigma_{t}^{2} & =2 \lambda^{2} r_{0}^{-5 / 3}\left[0.179 D^{-1 / 3}-0.145 d^{-1 / 3}\right]
\end{aligned}
$$

válido para $d \geq 2 D$, donde $D$ es la apertura del telescopio, $r_{0}$ es el parámetro de Fried y $\lambda$ es la longitud onda característica. Así, midiendo las varianzas es posible calcular $r_{0}$, de donde es posible obtener el seeing característico $\left(\varepsilon_{F W H M}\right)$ según:

$$
\varepsilon_{F W H M}=0.98 \frac{\lambda}{r_{0}} \quad\left(\operatorname{si}_{0} \ll D\right)
$$

para cada caso longitudinal y transversal.

\section{OBSERVACIONES}

Las observaciones fueron realizadas desde el Observatorio de Huancayo $(\mathrm{OH})\left(12^{\circ} 02^{\prime} 12^{\prime \prime}\right.$ latitud sur; $75^{\circ} 19^{\prime} 07^{\prime \prime}$ longitud oeste) durante siete noches consecutivas del 5 al 11 de agosto de 2012. Las condiciones atmosféricas durante las noches de observación fueron óptimas, es decir, no se observaron nubes pasajeras. El sistema utilizado que compone el OA-UNI DIMM incluye un telescopio Celestron de 8" equipado con una máscara con dos aberturas de $63.8 \mathrm{~mm}$ de diámetro cada una. Ambas aberturas están colocadas simétricamente a una distancia de $141.6 \mathrm{~mm}$ entre ellas.
Una cuña óptica con deflexión de 1', manufacturada por OFR (http://www.ofr.com) en vidrio óptico BK7, fue instalada en una de las aberturas como muestra la Fig. 2. El detector usando fue una cámara CCD WATEC 120N+ (Sony CCD ICX429ALL) facilitada por Bruno Sicardy del Observatorio de Paris (LESIA).El telescopio fue montado sobre el astrógrafo del $\mathrm{OH}$. Una computadora personal con procesador tipo Petium IV controló el uso de la cámara CCD.

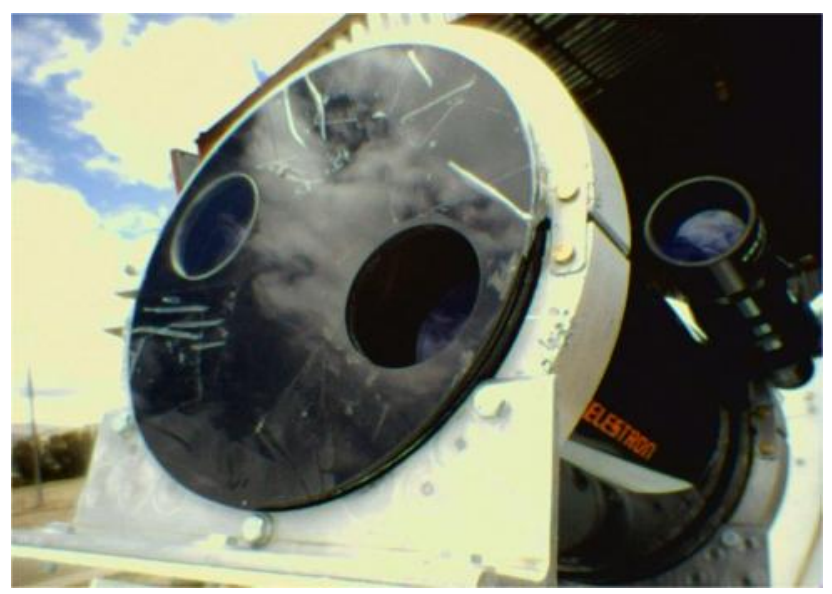

Fig. 2 Mascara con cuña óptica sobre el celestron 8.

La tasa de registro de un frame individual con el detector usado es de 0.11 segundos por frame. En cada frame son calculadas las posiciones de las dos imágenes generadas por el DIMM de la misma estrella. El cálculo es realizado a través del software "Seeing Monitor Analyser", el cual computa los centroides respectivos en el sistema de referencia del detector. Información adicional pertinente es también registrada en cada medida como la hora de la adquisición (en día juliano) y la masa de aire. Cada medida de seeing se calcula usando 50 frames consecutivos, lo que da un registro de seeing a cada 5.5 segundos. En cada frame las distancias relativas de los centroides de las dos imágenes en cada eje

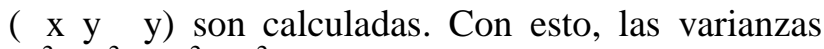
$\left(\sigma_{\mathrm{x}}^{2}=\sigma_{1}^{2}{ }_{1}\right.$ y $\left.\sigma_{\mathrm{y}}^{2}=\sigma_{\mathrm{t}}^{2}\right)$ a cada 50 frames son computadas y usando las ecuaciones (1) a (3), el seeing individual es obtenido. El promedio de los valores de seeing, longitudinal y transversal, es el mostrado en nuestros resultados. 
Las estrellas observadas con el DIMM fueron Achernar $(\alpha$ Eri) con magnitud visual V=0.50mag y coordenadas ecuatoriales (2000): ascensión recta (AR) 01:37:42.8, declinación (dec) -57:14:12.3; y,Altair $(\alpha$ Aql) con $\mathrm{V}=0.77$ y AR(2000) 19:50:47.0, $\operatorname{dec}(2000)$ 08:52:05.95. En general, Altair fue monitoreada durante la primera mitad de la noche y Achernar durante la segunda mitad.

\section{RESULTADOS}

La Tabla 1 muestra los resultados de las observaciones realizadas noche por noche con el OA-UNI DIMM en el OH en agosto del 2012. Se muestra el periodo observado (en horas) en cada noche, así como el número efectivo de medidas de seeing tomadas en el muestreo de cada noche. Finalmente, la estadística del seeing (en segundos de arco) para cada una de las siete noches es calculada. Ésta incluye el promedio, la mediana y el desvío padrón. Los valores mínimos obtenidos en cada noche también están indicados. La estadística considerando las medidas totales de las siete noches monitoreadas también es mostrada.

La primera noche con pocas horas observadas se debió al montaje inicial y calibración del sistema. La noche del 9 de agosto se presentó un problema técnico en el sistema eléctrico del observatorio por lo cual sólo se realizó medidas por 6 horas. A modo de ejemplo, en la Fig. 3 mostramos el monitoreo del seeing en el $\mathrm{OH}$ durante la noche del 06 de agosto. El gráfico muestra aproximadamente 3500 medidas de seeing durante las casi 7 horas de observación. Para facilitar la comparación, la Fig. 4 muestra el histograma de éstos valores de seeing, donde claramente se nota el pico centrado en 1.0".

El valor promedio considerando las siete noches es 1.34" con un desvío padrón de 0.33 ". La mejor noche corresponde al día 06 de agosto (analizada arriba) con un valor promedio de seeing de 1.03". El peor valor del seeing promedio es para el día 05 de agosto con 1.58". Considerando las medidas individuales durante toda la misión, el menor valor de seeing medido es de 0.5 ". En $14 \%$ del tiempo total monitoreado, el seeing fue menor que 1", y en $71 \%$, fue menor que 1.5 ". Comparativamente, los valores de seeing promedios en los mejores sitios de observación del planeta (Chile, Hawaii, Islas Canarias) se encuentran entre 0.5-0.8". A pesar, que los valores de seeing medidos durante esta misión observacional en el $\mathrm{OH}$ están lejos de los estándares de los observatorios profesionales, estimamos que el $\mathrm{OH}$ tenga un cielo de calidad mediana para las observaciones astronómicas. Con todo, son necesarias adicionales campañas de registro en diferentes meses del año para cuantificarlo mejor.

El presente trabajo ha mostrado la viabilidad de medidas usando la técnica DIMM, que por primera vez han sido realizadas y publicadas en Perú. Lugares adicionales en el territorio peruano con potencial para la observación astronómica serán posibles de calificar de manera padronizada usando la técnica mostrada.

En este sentido, una futura facilidad astronómica profesional será altamente beneficiada con medidas DIMM futuras.

Tabla 1. Log de las observaciones de seeing con OA-UNI DIMM en el OH (05-11/agosto/2012).

\begin{tabular}{ccccccccc}
\hline Noche & $\begin{array}{c}\text { Periodo } \\
\text { (horas) }\end{array}$ & Muestra & \multicolumn{6}{c}{ Seeing (") } \\
promedio & mediana & desvío padrón & min. & $<1$ " $(\%)$ & $<1.5 "(\%)$ \\
\hline 05 & 3 & 1614 & 1.58 & 1.53 & 0.34 & 0.74 & 1.8 & 46.5 \\
06 & 7 & 3491 & 1.03 & 1.01 & 0.21 & 0.49 & 47.7 & 96.9 \\
07 & 7 & 3526 & 1.33 & 1.28 & 0.31 & 0.65 & 12.0 & 73.4 \\
08 & 7 & 3007 & 1.46 & 1.42 & 0.29 & 0.70 & 2.9 & 60.7 \\
09 & 6 & 3243 & 1.44 & 1.38 & 0.35 & 0.62 & 6.7 & 61.3 \\
10 & 7 & 3686 & 1.40 & 1.37 & 0.27 & 0.64 & 4.4 & 67.3 \\
11 & 7.5 & 3920 & 1.32 & 1.26 & 0.30 & 0.68 & 11.6 & 77.3 \\
Todas & & 22502 & 1.34 & 1.30 & 0.33 & 0.49 & 13.6 & 71.4 \\
\hline
\end{tabular}




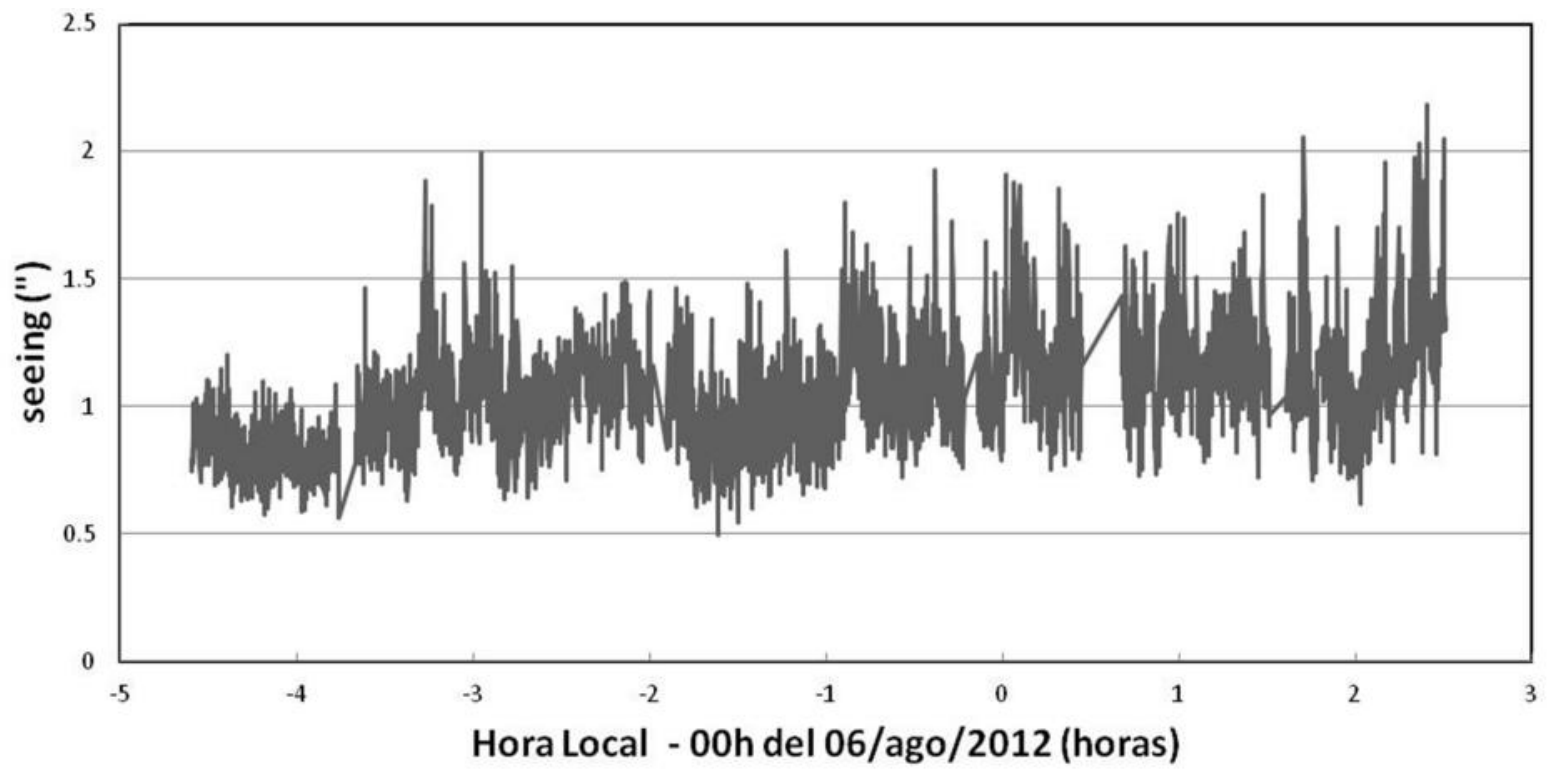

Fig. 3 Monitoreo del seeing durante la noche del 06/ago/2012 en el OH.

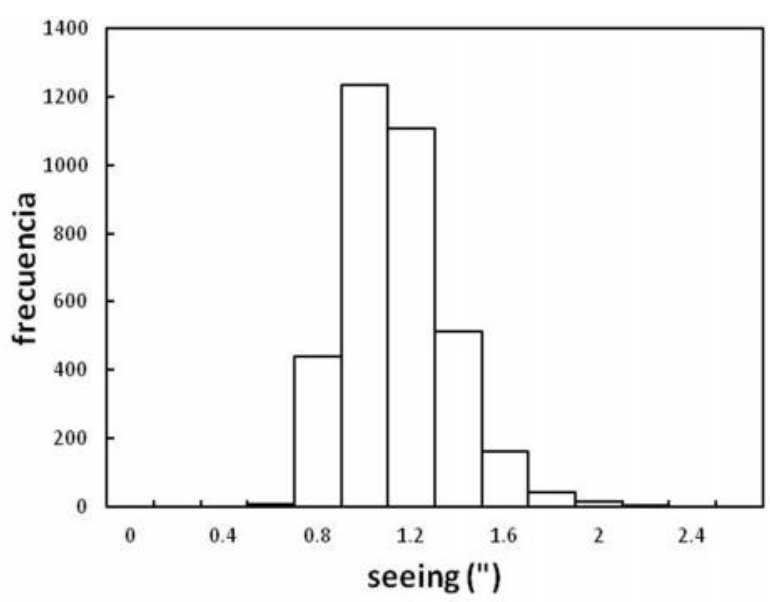

Fig. 4 Histograma de los valores del seeing para la noche del 06 de agosto del 2012.

\section{CONCLUSIONES}

En este trabajo, se realizaron las primeras medidas de seeingcon el OA-UNI DIMM usando la técnica de monitoreo del movimiento diferencial de imagen en el Observatorio de Huancayo. El dispositivo óptico respondió a las expectativas de manera satisfactoria. Fue monitoreado el seeing durante siete noches consecutivas en el $\mathrm{OH}$ cubriendo en promedio intervalos de 6-7 horas en cada noche.
El valor promedio durante las noches monitoreadas fue de $1.34 " \pm 0.33 "$. El mejor valor promedio de seeing obtenido para una única noche fue de 1.03 " \pm 0.21 ”, en la segunda jornada de observación. Buenos valores de seeing ( $<1$ ") fueron medidos en $14 \%$ del tiempo monitoreado. Por otro lado, valores razonables de seeing $(<1.5$ ") estuvieron presentes en $71 \%$ del tiempo observado.

En base, a las medidas presentadas en este trabajo el $\mathrm{OH}$ califica como un cielo de mediana calidad para observaciones astronómicas. Con todo, medidas adicionales son necesarias para cuantificar mejor la variación estacional del seeing en el $\mathrm{OH}$. Especial atención debe ser tomada para las épocas de lluvia en la sierra andina, de modo a verificar la viabilidad de las medidas. Adicionalmente, la cuantificación de la polución luminosa de las ciudades cercanas debe ser mensurada para determinar su real impacto en las medidas astronómicas en el $\mathrm{OH}$.

\section{REFERENCIAS}

1. Rösch, Radium, "Journal of Physic", pp. 15, 39 S, 1954.

2. Keller, S. J., Keller, G. "Astronomical Seeing, de Telescopes", Vol. 1, Chicago, Chicago Univ. 
Press, 1960, pp. 138 - 153.

3. Meinel, "Astronomical Seeing and Observatory Site Selection de Telescopes", Vol. I, Chicago, Chicago Univ. Press, 1960, pp. 154 - 175.

4. Tatarski, "Wave Propagation in a Turbulent Medium", New York: Dover, 1961.

5. Roddier, "The Effects of Atmospheric Turbulence in Optical Astronomy", Progress in Optics, pp. 281 - 373, 1981.

6. Fried, "Statistics of a Geometric Representation of Wave front Distortion", Journal of the Optical Society of America, pp. 1427, 1965.

7. Young, "Seeing: its Cause and Cure", Astrophysical Journal, pp. 587-604, 1974.

8. Roddier, F., Lena, P., "Long-baseline Michelson interferometry with large groundbased telescopes operating at optical wavelengths. I - General formalism: Interferometry at visible wavelengths", JOSA, pp. 171-182, 1986.

9. Fort, D., "Multi - object spectroscopy with the
10. Azouit, M., Vernin, J., "Remote investigation of tropospheric turbulence by two-dimensional analysis of stellar scintillation", Journal of Atmospheric Science, Vol. 37, pp. 1550 - 1557, 1980.

11. Sarazin, M., Roddier, F., "The ESO differential imaging motion monitor", Astronomy and Astrophysics , pp. 294-300, 1990.

12. Martin, F., Tokovinin, A., Agabi, A., Borgnino, J., Ziad, A., "G.S.M.: a Grating Scale Monitor for atmospheric turbulence measurements. I. The instrument and first results of angle of arrival measurements", Astronomy and Astrophysics, pp. 173-180, 1994.

Correspondencia: emezaq@uni.pe

Recepción de originales: abril 2013

Aceptación de originales: diciembre 2013
VLT", de Second Workshop on ESO's Very Large Telescope, Proceedings, Venice- Italy, 1986. 